\title{
Stress dielectric response in liquid polymers
}

\author{
Yiyan Peng, Yuri M. Shkel, ${ }^{\text {a) }}$ and GeunHyung Kim \\ Department of Mechanical Engineering, University of Wisconsin, Madison, \\ Wisconsin 53706
}

(Received 20 July 2004; final revision received 19 October 2004)

\begin{abstract}
Synopsis
Deformation-induced dielectric response of dielectric materials, called dielectrostriction, provides a new approach to study properties and structure of liquid polymers. The dielectrostriction effect resembles the well-known birefringence phenomenon. While birefringence in liquid polymers is described by the stress-optic relationship, a stress-dielectric relationship applies to dielectrostriction. However, dielectrostriction measurements can be performed on both transparent and opaque materials, require a much simpler data acquisition technique, are capable of local measuring stresses, and can be implemented for in-line monitoring of polymer processing. In this study, a planar capacitor sensor technique has been developed to detect the dielectrostriction effect in shear flow of liquid polymers. Experimental evidences of the dielectrostriction effect and the stress-dielectric relationship in liquid polymers are presented for both silicone elastomer and poly $(\epsilon$-caprolactone). Mechanisms contributing to the similarity between the stress-dielectric and the stress-optic relationship are discussed. (C) 2005 The Society of Rheology.
\end{abstract}

[DOI: $10.1122 / 1.1835340]$

\section{INTRODUCTION}

Rheo-optical measurements are traditionally employed to study flow of polymeric fluids. Flow-induced birefringence provides valuable information about the rheology and microstructure of polymers because both stress and birefringence are similarly related to an orientation distribution of the chain segments [Bird et al. (1987)]. Optical measurements can be applied to a localized volume of a material without mechanical contact with the flowing medial. This makes the rheo-optical method an important addition to rheological instrumentation [Janeschitz-Kriegl (1983), Saiz and Rainde (1992), Wales (1976)]. However, such measurements can be conducted only with transparent materials, require complicated and bulky optical setups, and are unsuitable for in-line implementation.

Dielectrostriction, which is a previously overlooked rheological phenomenon, is free of many shortcomings of optical techniques and provides new avenues to study liquid polymers. Dielectrostriction is defined here as a variation of dielectric properties of the material with deformations and has a physical basis very similar to that of the rheooptical measurements - birefringence. Moreover, flow-induced birefringence can be considered as a manifestation of the dielectrostriction in the optical range of electromagnetic spectrum. While birefringence obeys a stress-optic rule (SOR) [Janeschitz-Kriegl (1983), Saiz and Rainde (1992), Wales (1976)], the stress-dielectric rule (SDR) should be appli-

\footnotetext{
a) Author to whom all correspondence should be addressed; electronic mail: yshkel@engr.wisc.edu
} 
cable to the dielectrostriction phenomenon. However, while only electronic polarization is involved in the birefringence, all polarization mechanisms [e.g., interfacial (MaxwellWagner), dipole, ionic, or electronic polarization mechanisms] contribute to the dielectrostriction. Although optical measurements are limited to transparent materials, dielectrostriction is applicable to both transparent and opaque materials. Dielectrostriction measurements can be efficiently applied to suspensions where the flow induced birefringence is difficult to observe. In addition, dielectrostriction measurements can be conducted over a wide range of electric field frequencies, including those where particular specie of the suspension has the strongest response. Moreover, according to Shkel and Klingenberg (1998), the dielectrostriction response of a suspension is determined by the pair-distribution function of the suspended particles. Therefore, one can speculate that the dielectrostriction measurements are capable of providing valuable information about the material microstructure.

The dielectrostriction is converse effect of a phenomenon called electrostriction. According to Stratton (1941), electrostriction is defined as a material's deformation due to electrically-induced stresses. Following Landau, Lifshitz, and Pitaevskii (1984), fieldinduced stress, $\sigma_{i k}$, is related to the dielectric displacement, $\mathbf{D}$, and the electric field, $\mathbf{E}$, as

$$
\sigma_{i k}=\sigma_{i k}^{\text {mech }}-\left.\frac{1}{2} \frac{\partial D_{l}}{\partial u_{i k}}\right|_{T, E} \cdot E_{l}-\frac{\mathbf{E} \cdot \mathbf{D}}{2} \delta_{i k}+\frac{E_{i} D_{k}+E_{k} D_{i}}{2} .
$$

Here the total stress, $\sigma_{i k}$ is composed of the mechanical stress, $\sigma_{i k}^{\text {mech }}$, which would be the total stress in the absence of an electric field, electrostriction stress, $-1 / 2 \partial D_{l} /\left.\partial u_{i k}\right|_{T, E} \cdot E_{l}$ and the Maxwell's stress, $-\mathbf{E} \cdot \mathbf{D} \delta_{i k} / 2+\left(E_{i} D_{k}+E_{k} D_{i}\right) / 2$. Material parameters describing the effect of strain on polarization, $\partial D_{l} / \partial u_{i k}$, are the electrostriction parameters. Equation (1) is valid for any material, including those which are anisotropic. Stratton (1941) had previously offered an expression for isotropic materials which is similar to Eq. (1). One can obtain the field-induced stress in any polarizable material (electrostriction) by determining the strain dependence of the dielectric displacement in the material, $\partial D_{l} /\left.\partial u_{i k}\right|_{T, E}$, (dielectrostriction). Following such a procedure, Shkel and Klingenberg (1999) derived the total electrically-induced stress in uniaxially isotropic composite materials. Anderson (1986) used a somewhat equivalent procedure to calculate the stress in a cubic composite which is electrically excited in the lattice direction. Thus the dielectrostriction phenomenon is intrinsically related to material's electroactivity.

Simultaneously monitoring deformation of the dielectric layer between two parallel electrodes and the overall capacitance of the same electrodes is one way to measure the dielectrostriction effect. This technique has been applied by Shkel and Klingerberg (1996) to evaluate the electrostriction parameters for two polyurethane films and by Shkel et al. (2002) to study the silicone rubber composites having different structures. The high sensitivity of the dielectrostriction measurements to microstructure of the tested material has been observed. The present article adopts another sensing configuration where interdigitated electrodes are deposited on a surface adjacent to the deformed dielectric material. Such a planar configuration can be easily located on the surface of a wall and is, therefore, more suitable for in-line monitoring of polymeric flows than are parallel capacitor sensors. A sensor of such planar configuration was utilized previously to study the shear dielectric response of solid materials [Filanc-Bowen et al. (2003)].

This article discusses the dielectrostriction phenomenon in oscillatory shearing liquid polymers. Section II, introduces the theoretical background of shear dielectrostriction. 
TABLE I. Comparison of predicted dielectrostriction coefficients for (a) fluids [Stratton (1941)], (b) isotropic solids [Shkel and Klingenberg (1998)], and (c) solids having cubic symmetry [Anderson (1986)], all materials have the same dielectric constant. (d) Dielectrostriction response of a cubic material requires the third coefficient, $\alpha_{3}$ [Nye (1985)], whose predictions are not currently available. (NA stands for not applicable.)

\begin{tabular}{lcccc}
\hline \hline Material & $\begin{array}{c}\text { Dielectric } \\
\text { constant }\end{array}$ & $\alpha_{1}$ & $\alpha_{2}$ & $\alpha_{3}$ \\
\hline Newtonian fluid $^{(\mathrm{a})}$ & $\epsilon$ & 0 & $-\frac{1}{3}(\epsilon-1)(\epsilon+2)$ & 0 \\
Isotropic solid $^{(\mathrm{b})}$ & $\epsilon$ & $-\frac{2}{5}(\epsilon-1)^{2}$ & $-\frac{1}{3}(\epsilon-1)(\epsilon+2)+\frac{2}{15}(\epsilon-1)^{2}$ & 0 \\
Cubic solid $^{(\mathrm{c})}$ & $\epsilon$ & $-1.515(\epsilon-1)^{2}$ & $-\frac{1}{3}(\epsilon-1)(\epsilon+2)+0.505(\epsilon-1)^{2}$ & $\mathrm{NA}^{(\mathrm{d})}$ \\
\hline \hline
\end{tabular}

Phenomenology of the dielectrostriction and the stress-dielectric rule are formulated for general deformations. The background of planar capacitor sensors for monitoring shear flow of liquid polymers is provided. The stress-dielectric rule is formulated and verified experimentally for oscillatory shear flows of liquid silicone elastomer and $\operatorname{poly}(\epsilon-$ caprolactone) (PCL) in Sec. III. The results are discussed and the main conclusions of this article are summarized in Sec. IV. The present study indicates that dielectrostriction measurements are capable of becoming a valuable analytical tool for studying liquid viscoelastics.

\section{THEORETICAL BACKGROUND}

\section{A. Phenomenology of dielectrostriction}

\section{Dielectrostriction}

Dielectric properties of deformed isotropic dielectrics are described by a second order tensor, $\epsilon_{i j}$, which can be approximated as a linear function of the strain tensor, $u_{i j}$. Three material parameters, the relative dielectric constant of the undeformed material, $\epsilon$, and two electrostriction parameters, $\alpha_{1}$ and $\alpha_{2}$, are needed to describe the dielectrostrictive properties of an isotropic material [Landau, Lifshitz, and Pitaevskii (1984), Shkel and Klingenberg (1998), Stratton (1941)]:

$$
\Delta \epsilon_{i j}=\left(\epsilon_{i j}-\epsilon \delta_{i j}\right)=\alpha_{1} u_{i j}+\alpha_{2} u_{k k} \delta_{i j} .
$$

Parameter $\alpha_{1}$ is responsible for the changes in dielectric properties of the material due to a linear deformation, while $\alpha_{2}$ accounts for the volume changes. Parameters $\alpha_{1}$ and $\alpha_{2}$ can be determined from two measurements in which $u_{k k} / u_{i j}$ differs [Shkel and Klingenberg (1996)]. The strain tensor is related to the displacement field $\mathbf{U}\left(x_{1}, x_{2}, x_{3}\right)$ [Mase and Mase (1999)] as

$$
u_{i j}=\frac{1}{2}\left[\frac{\partial U_{i}}{\partial x_{j}}+\frac{\partial U_{j}}{\partial x_{i}}+\frac{\partial U_{k}}{\partial x_{i}} \frac{\partial U_{k}}{\partial x_{j}}\right] .
$$

Only the first two terms of Eq. (3) are traditionally retained for small deformations. However, the contribution of these two terms to shear dielectrostriction is quadratic with strain. The nonlinear term therefore should be also taken into account.

Dielectrostriction measurements are very sensitive to material microstructure. According to Shkel and Klingenberg (1998), the dielectrostriction response of a suspension is determined by the pair-distribution function of the suspended particles. Even materials showing the same dielectric properties, determined by a single dielectric constant, $\epsilon$, will provide vastly different results for $\alpha_{1}$ and $\alpha_{2}$, see Table I. In a static situation, both isotropic liquids and solids might have a similar random structure and the same dielectric 
constants. However, fluids remain isotropic during any flow-induced deformation and the coefficient $\alpha_{1}$ therefore is always zero. The only dielectrostriction effect observed in Newtonian fluids and gases is due to the variations of the dielectric constant with the density. Materials having cubic structure are traditionally considered as a lattice approximation for isotropic materials. However, the description of the photoelastic effect in such materials requires three coefficients, see Nye (1985), p. 252. Due to the existing analogy between the photoelastic and dielectrostriction effects, the dielectrostriction response of cubic materials also requires the third coefficient, $\alpha_{3}$, microscopic models for which are not yet available. (Following the notation used here, the electrostriction coefficient in cubic materials can be introduced as $\epsilon_{i j}=\epsilon \delta_{i j}+\alpha_{1} u_{i j}+\alpha_{2} u_{k k} \delta_{i j}+\alpha_{3} u_{l m} d_{l} d_{m} d_{i} d_{j}$, where $d_{i}$ is the unit vector in the lattice direction.) Dielectrostriction behavior of anisotropic materials is even more complicated than that under isotropy. The general phenomenological approach to dielectrostriction in anisotropic solids is outlined by Shkel and Klingenberg (1999). The dielectrostriction effect manifests itself differently in liquids and solids. However, polymeric liquids exhibit both viscous and elastic deformations [see, for example, Bird et al. (1987)]. A variation in the structure of liquid polymers with a linear deformation is very similar to that of solids and can cause the pronounced dielectrostriction effect, thereby rendering the dielectrostriction measurements effective for liquid polymers.

\section{SDR}

The SDR in liquid polymers can be formulated similarly to the SOR in ideal elastic dielectrics. Under small deformations, both liquid and solid elastic materials demonstrate a linear relationship between the total stress, $\sigma_{i k}$, and the strain, $u_{l m}$. For an oscillatory shear deformation, the total stress at an instantaneous moment can be decomposed into two stresses, $\sigma_{i k}=\sigma_{i k}^{\prime}+\sigma_{i k}^{\prime \prime}$ : elastic stress, $\sigma_{i k}^{\prime}$, which is in phase with the deformation, $u_{i k}$, and viscous stress, $\sigma_{i k}^{\prime \prime}$, which is out of phase with the deformation. For shear deformations, $u_{12}$, one can introduce the storage modulus, $G^{\prime}=\sigma_{12}^{\prime} / u_{12}$, and the loss modulus, $G^{\prime \prime}=\sigma_{12}^{\prime \prime} / u_{12}$. For the elastic contribution to the total mechanical stress, one can write

$$
\sigma_{i k}^{\prime}=\frac{E_{Y}^{\prime}}{1+\nu}\left(u_{i k}+\frac{\nu}{1-2 \nu} u_{l l} \delta_{i k}\right) \quad \text { and } G^{\prime}=\frac{E_{Y}^{\prime}}{2(1+\nu)},
$$

where $\nu$ is the Poisson's ratio. This is the most general linear relationship between the stresses and the strains in isotropic materials. In fact, this is the Hooke's Law if $E_{Y}^{\prime}$ is assumed to be a frequency-dependent effective elastic modulus. Equation (4) can be rewritten for the strains in terms of the stresses

$$
u_{i k}=\frac{1+\nu}{E_{Y}^{\prime}} \sigma_{i k}^{\prime}-\frac{\nu}{E_{Y}^{\prime}} \sigma_{l l}^{\prime} \delta_{i k} .
$$

One can assume that the dielectrostriction is related to the strains as described in Eq. (2). Combining Eqs. (2) and (5) gives

$$
\epsilon_{x x}-\epsilon_{y y}=C^{\prime}\left(\sigma_{x x}^{\prime}-\sigma_{y y}^{\prime}\right), \quad \epsilon_{y y}-\epsilon_{z z}=C^{\prime}\left(\sigma_{y y}^{\prime}-\sigma_{z z}^{\prime}\right), \quad \epsilon_{x x}-\epsilon_{y y}=C^{\prime}\left(\sigma_{x x}^{\prime}-\sigma_{y y}^{\prime}\right),
$$

where $C^{\prime}=\alpha_{1}(1+\nu) / E_{\gamma}^{\prime}$. The shear dielectrostriction is expressed as

$$
\epsilon_{x y}=C^{\prime} \sigma_{x y}^{\prime}, \quad \epsilon_{y z}=C^{\prime} \sigma_{y z}^{\prime}, \quad \epsilon_{z x}=C^{\prime} \sigma_{z x}^{\prime} .
$$




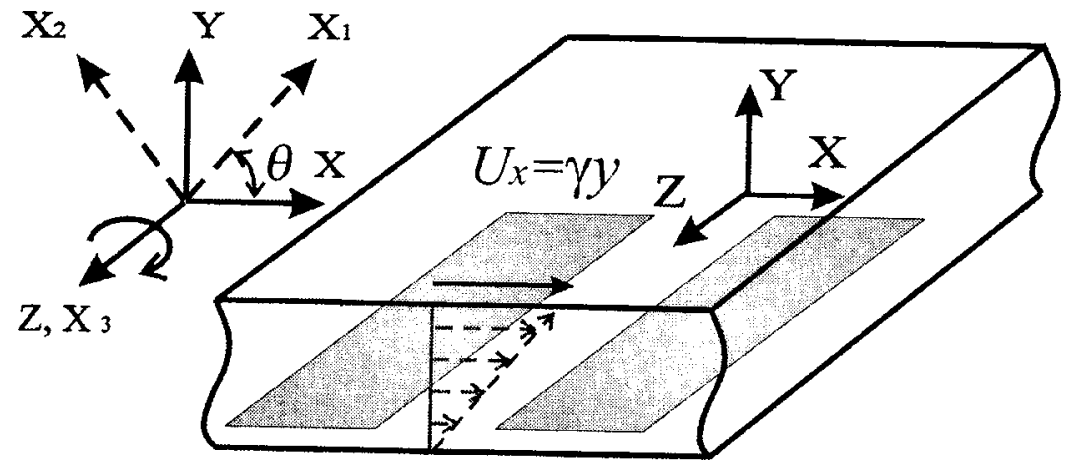

FIG. 1. An initially isotropic material is subjected to a shear displacement, $U_{x}=\gamma y$, perpendicular to the electrode strips.

If one recognizes that the magnetic permeability of polymers, $\mu$, is close to one $(\mu \approx 1)$ and replaces $\epsilon$ with $\sqrt{n}$ (note $n^{2}=\epsilon \mu$ ), these expressions resemble the well-known stress-optic rule and are defined here as "stress-dielectric rule." Resemblance will be even closer if one recognizes the relation $\Delta n_{i k} / n=\Delta \epsilon_{i k} / 2 \epsilon$, which is valid for small deformations.

\section{B. Planar capacitor sensor}

A planar capacitor sensor with interdigitated electrodes is implemented to detect the dielectrostriction response of liquid polymers to an oscillatory shear flow. The flow causes a change in the dielectric constant of the polymer, and, therefore, the capacitance of the system of electrodes. The following is a detailed examination of rheological and electronic aspects of the dielectrostriction measurements.

\section{Dielectric response due to shear deformation}

An initially isotropic material is subjected to a simple shear displacement, $U_{x}$

$=\gamma y$, perpendicular to the electrode strips as shown in Fig. 1. This yields nonzero components of the strain tensor defined in the $X Y Z$ coordinate system

$$
u_{x y}=u_{y x}=\frac{\gamma}{2} \text { and } u_{y y}=\frac{\gamma^{2}}{2} \text {. }
$$

The principal coordinate system, $X_{1} X_{2} X_{3}$, can be obtained by rotating the original $X Y Z$-coordinate system through an angle $\theta$ around the $Z$ axis,

$$
\theta=\arctan \left(\frac{\gamma}{2} \pm \sqrt{1+\frac{\gamma^{2}}{2}}\right)
$$

In the principal coordinate system, the simple shear deformations are equivalent to superposition of tensile and compressive strains defined by their principal values

$$
u_{1}=\frac{\gamma^{2}}{4}+\frac{\gamma}{4} \sqrt{\gamma^{2}+4}, \quad u_{2}=\frac{\gamma^{2}}{4}-\frac{\gamma}{4} \sqrt{\gamma^{2}+4} \quad \text { and } u_{3}=0 .
$$

Note, for pure shear deformations, when $u_{y y}=0$, Eqs. (9) and (10) yield a well-known result $\theta= \pm 45^{\circ}, u_{1}=\gamma / 2, u_{2}=-\gamma / 2$, and $u_{3}=0$. 


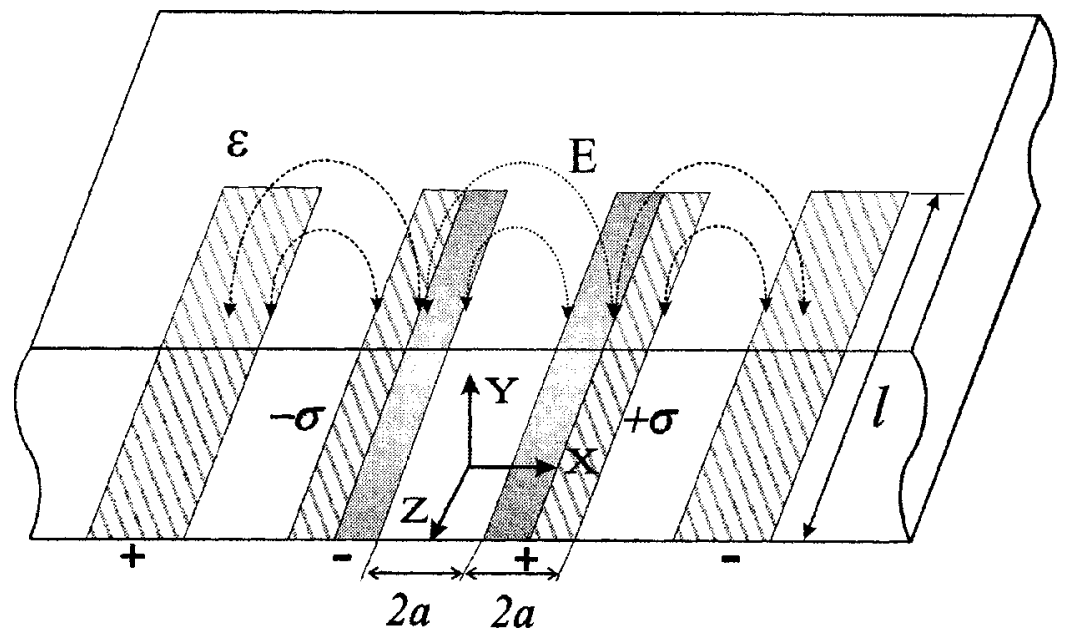

FIG. 2. Electrodes of equal width, $2 a$, and total length, $L$, are separated by $2 a$. Deformation of the dielectric layer affects dielectric constant of the material and, therefore, the sensor's capacitance.

According to Eq. (2), coordinate system $X_{1} X_{2} X_{3}$ is also the principal coordinate system for dielectric tensor of the deformed material. The principal dielectric constants are therefore

$$
\begin{gathered}
\epsilon_{1}=\epsilon+\alpha_{1}\left(\frac{\gamma^{2}}{4}+\frac{\gamma}{4} \sqrt{\gamma^{2}+4}\right)+\alpha_{2} \frac{\gamma^{2}}{2}, \quad \epsilon_{2}=\epsilon+\alpha_{1}\left(\frac{\gamma^{2}}{4}-\frac{\gamma}{4} \sqrt{\gamma^{2}+4}\right)+\alpha_{2} \frac{\gamma^{2}}{2}, \\
\epsilon_{3}=\epsilon+\alpha_{2} \frac{\gamma^{2}}{2} .
\end{gathered}
$$

\section{Concept of planar capacitor sensor}

A planar capacitor sensor, Fig. 2, detects deformations through changes in dielectric properties of material near the interdigitated electrodes. All electrodes have equal width, $2 a$, are equally separated by $2 a$. Both the thickness of the dielectric layer, $h$, and the length of the electrodes, $l$, are much larger than the distance between the electrodes $(h / 2 a, l / 2 a \gg 1)$. Deformation of the dielectric layer affects the material's dielectric constant and, therefore the capacitance of the electrodes.

(a) Capacitance of interdigitated electrodes. Capacitance, $C$, of any system of electrodes is defined as the ratio

$$
C=\frac{Q}{V},
$$

where $Q$ is the charge needed to produce the potential difference, $V$. Surface density of the charge, $\kappa$, can be expressed through the normal component of the dielectric displacement, $D_{n}$, on the electrodes

$$
\kappa=D_{n 1}+D_{n 2} .
$$

The dielectric displacements, $D_{n 1}$, and, $D_{n 2}$, are taken at the same locations but on the opposite sides of the electrode surfaces. 


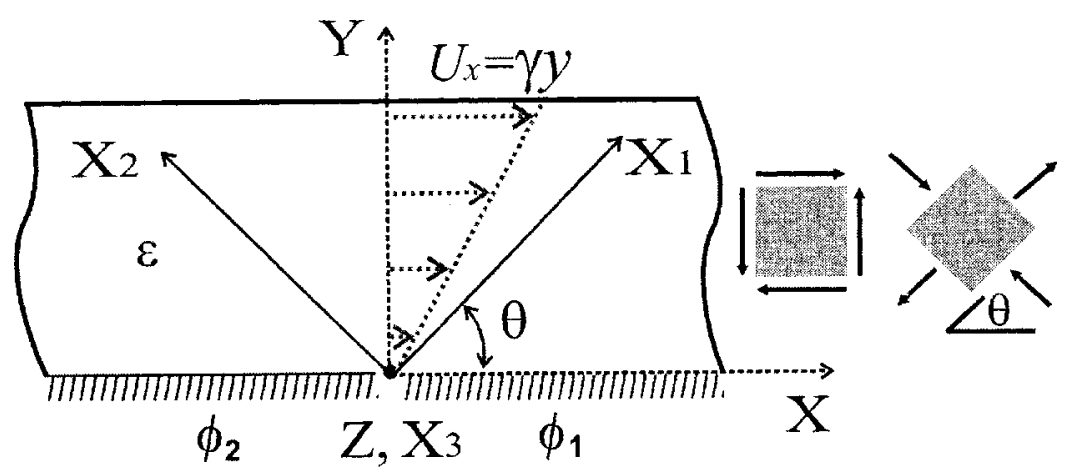

FIG. 3. Two electrodes, each covering half of the plane, are very close to each other and parallel to the axis $Z$. One electrode is under electrical potential $\phi_{1}$ and the other under $\phi_{2}$. A material with dielectric constant, $\epsilon$, on top of the electrodes is subjected to shear deformation, $\gamma$. Square element on the left depicts stresses in the original, $X Y Z$, coordinate system and square element on the right depicts stresses in the principal, $X_{1} X_{2} X_{3}$, coordinate systems.

Two sets of electrodes shown in Fig. 2 form a planar capacitor sensor. Potential difference, $V$, is applied across the even and odd electrodes. The capacitance of this electrode configuration can be calculated numerically. However, it would be instructional to first consider a electrode configuration in Fig. 3 which allows for the exact analytical solution. Two electrodes, each covering half of the plane, are almost touching each other along the axis $Z$. One electrode has the electrical potential, $\phi_{1}$, and the other has the potential $\phi_{2}$. The overall electrode length, $L$, is equal to the total length of all individual strips in Fig. 2. An isotropic material having dielectric constant, $\epsilon$, is located on the top of the electrodes.

The electrode strip having length, $L$, and width, $d r$, carries charge, $d Q$ :

$$
d Q=\kappa L d r .
$$

The dielectric displacement, $\mathbf{D}$, of an isotropic dielectrics having the dielectric constant, $\epsilon$, in presence of the electric field, $\mathbf{E}$, is $\mathbf{D}=\epsilon_{0} \epsilon \mathbf{E}$, where $\epsilon_{0}$ is the dielectric constant of free space. The field can be obtained in a straightforward manner by solving Laplace's equation, $\nabla^{2} \phi=0$, where $\mathbf{E}=-\nabla \phi$. However, an originally isotropic material becomes anisotropic if subjected to a shear deformation and its dielectric properties are described by the second rank tensor, $\boldsymbol{\epsilon}$. The dielectric displacement, $\mathbf{D}$, is, therefore, related to the applied field as

$$
\mathbf{D}=\epsilon_{0} \boldsymbol{\epsilon} \cdot \mathbf{E}
$$

The equation for the potential, $\phi$, becomes $\nabla \cdot \boldsymbol{\epsilon} \cdot \nabla \phi=0$, which is simpler in the principal coordinate system, $X_{1} X_{2} X_{3}$ :

$$
\epsilon_{1} \frac{\partial^{2} \phi}{\partial X_{1}^{2}}+\epsilon_{2} \frac{\partial^{2} \phi}{\partial X_{2}^{2}}+\epsilon_{3} \frac{\partial^{2} \phi}{\partial X_{3}^{2}}=0 .
$$

Here the dielectric tensor, $\boldsymbol{\epsilon}$, has a diagonal form with the principal dielectric constants, $\epsilon_{1}, \epsilon_{2}$, and $\epsilon_{3}$ given by Eq. (11). Note that this last equation becomes Laplace's equation only if $\epsilon_{1}=\epsilon_{2}=\epsilon_{3}=\epsilon$. However, Eq. (16) can be converted into Laplace's equation by scaling the coordinate axes $X_{1}^{\prime}=\sqrt{\epsilon_{1}} X_{1}, X_{2}^{\prime}=\sqrt{\epsilon_{2}} X_{2}$ and $X_{3}^{\prime}=\sqrt{\epsilon_{3}} X_{3}$. Equation (16) can be easily solved in the coordinate system $X_{1}^{\prime} X_{2}^{\prime} X_{3}^{\prime}$ and the obtained solution then 
converted back to the coordinate system $X_{1} X_{2} X_{3}$. The potential of the electric field in the original, $X_{1} X_{2} X_{3}$, coordinate system is

$$
\phi=\phi_{1}+\frac{\phi_{2}-\phi_{1}}{\pi}\left(\tan ^{-1} \sqrt{\frac{\epsilon_{1}}{\epsilon_{2} X_{2}}}+\tan ^{-1} \frac{\gamma+\sqrt{\gamma^{2}+4}}{2} \sqrt{\frac{\epsilon_{1}}{\epsilon_{2}}}\right) .
$$

The normal component of the dielectric displacement at the electrode surfaces obtained from the field potential and Eq. (15) is

$$
D_{n}=\frac{\epsilon_{0} \sqrt{\epsilon_{1} \epsilon_{2}}}{\pi r}\left(\phi_{1}-\phi_{2}\right)
$$

where $r$ is the distance from the origin of the coordinate system to a point on the electrode surface. The principal values of $\epsilon_{1}$ and $\epsilon_{2}$ are given by Eq. (11).

Once $D_{n}$ is known, the charge density at the electrode surface, $\kappa$, follows from Eq. (13). Consequently the total charge, $Q$, can be obtained by integrating the charge distribution over the entire electrode surface. We will assume that the solution given by Eq. (18) is also valid for the dielectric displacement at the electrodes of the sensor in Fig. 2. Due to symmetry of the field distribution near the electrodes, the whole system can be modeled as just two electrodes of the width, $a$, and the total length, $L$. Finally the capacitance, $C$, is determined from Eq. (12) as the ratio between the electrodes charge and the potential difference, $V=\phi_{1}-\phi_{2}$ :

$$
C=\frac{1}{V} \int_{a}^{2 a} d Q=\frac{\ln 2}{\pi} \epsilon_{0}\left(\sqrt{\epsilon_{1} \epsilon_{2}}+\epsilon_{s}\right) L .
$$

Here $\epsilon_{s}$ is the dielectric constant of the substrate to which the electrodes are attached. The exact solution for the capacitance, $C$, will provide us with a correction to the coefficient $\ln 2 / \pi$ in Eq. (19), which, however, will be cancelled in the final expressions.

(b) Variation of capacitance with deformation. The sensor capacitance before deformation, $C_{0}$, is given by Eq. (19) where $\epsilon_{1}=\epsilon_{2}=\epsilon$. The deformation affects the capacitance, $C$, which would be given by the same Eq. (19) but with $\epsilon_{1}$ and $\epsilon_{2}$ determined from Eq. (11). Deformation of the dielectric material changes the principal values of the dielectric tensor, $\epsilon_{1}$ and $\epsilon_{2}$. Only the quadratic terms of the shear strains, $\gamma$, should be retained in the final expression for the variation of the capacitance

$$
\frac{\Delta C}{C_{0}}=\frac{C-C_{0}}{C_{0}}=\frac{2 \epsilon \alpha_{1}-\alpha_{1}^{2}+4 \epsilon \alpha_{2}}{8 \epsilon^{2}} \gamma^{2} .
$$

The resulting variations of the capacitance can be measured by a number of wellestablished methods using either off-the-shelf or custom-made instrumentation [Doeblin (1990), Holman (1994), Horowitz and Hill (1998)].

\section{EXPERIMENTAL STUDIES}

\section{A. Experimental setup}

A schematic of the experimental setup is shown in Fig. 4. An AR-1000 rheometer from TA Instruments is used to provide the oscillatory shear flow between two concentric cylinders: the external cylindrical container (having inner radius $R_{2}=22.5 \mathrm{~mm}$ ) is fixed and the inner cylinder (having radius $R_{1}=21 \mathrm{~mm}$ and height $H=8 \mathrm{~mm}$ ) is rotated by the rheometer. The liquid to be tested is placed in the gap between the two cylinders. 


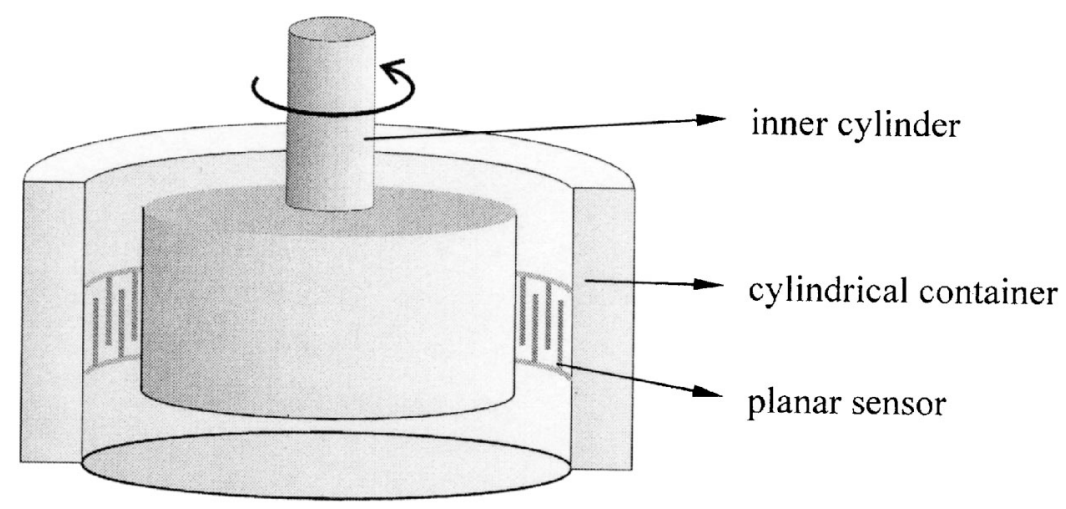

FIG. 4. Planar capacitor sensor is attached to the inner surface of the cylindrical container; rotation of the inner cylinder produces shear flow.

Since the gap between the cylinders is much less than their radii, the lubrication approximation can be applied to the flow description. The rheometer output signal is also used to compare flow-induced stresses and the dielectric response of the samples. The previously described planar capacitor sensor is attached to the inner surface of the external cylinder. Shear flow due to the oscillating inner cylinder causes changes in the dielectric properties of the sample and, consequently, the changes in capacitance of the sensor, $C_{x}$. The electronic circuitry of Fig. 5 is used to measure the small variation in the capacitance [Doeblin (1990), Holman (1994), Horowitz and Hill (1998)]. The voltage across the sensor electrodes is fixed, $V=$ const, and the variation in the capacitance produces a current flow across the high impedance resistor, $R$. A voltage variation due to the dielectrostriction response is amplified, filtered and then measured by a SR830 DSP lock-in amplifier.

The flow profile due to oscillations of the inner cylinder is approximated by dividing the fluid into two regions which are depictured as in Fig. 6(a): (1) plane flow in $d_{1}$ $=1.5 \mathrm{~mm}$ gap between the inner cylinder and the bottom surface of the cylindrical container; and (2) circular annular Couette flow in the $d_{2}=1.5 \mathrm{~mm}$ gap between the inner and the outer cylinders.

Since the measured dielectrostriction signal is due to the deformation of a localized volume near the electrodes, only the shear strain/stress at the sensor location should be related to the dielectrostriction signal. However, the rheometer records the total torque, $M_{\text {total }}$, which is the contribution from both regions (1) and (2), see Fig. 6(a). An additional step is needed for each test to obtain torque, $M_{2}$, due to the circular annular

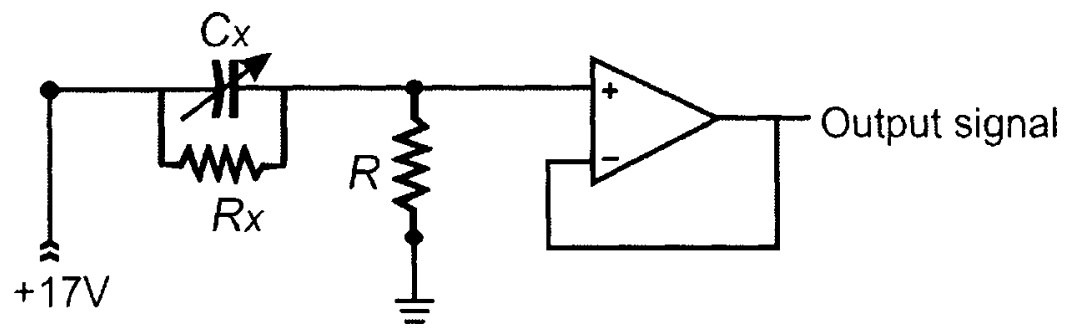

FIG. 5. Variable capacitor, $C_{x}$, and resistor, $R_{x}$, represent the sensor and the sample. Voltage due to the current through resistor, $R$, is amplified, filtered and measured. 
(a)

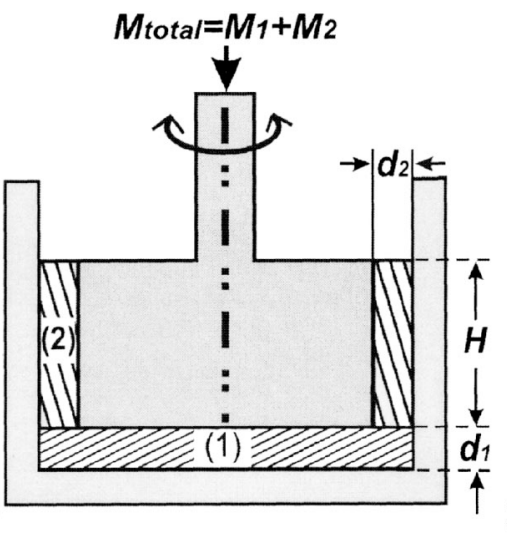

(b)

FIG. 6. (a) Flows in both regions 1 and 2 contribute to the total torque $M_{\text {total }}$; (b) torque $M_{1}$ is due to the flow in region 1 alone.

Couette flow in the region (2). In this step, the sample being tested occupies only the region (1), Fig. 6(b). Torque $M_{1}$ from the flow in the gap between the inner cylinder and the bottom surface of the cylindrical container is recorded. The torque due to the shear flow in the region (2) can be determined as $M_{2}=M_{\text {total }}-M_{1}$. Consequently, the corresponding shear stress near the electrodes, $\sigma=F_{\sigma} M_{2}$, can be calculated, where the shear stress factor $F_{\sigma}=1 / 2 \pi H R_{2}^{2}$ is determined by the geometry.

\section{B. Testing procedure}

A thermoset polymer, silicone elastomer (GI-1100 from Silicones, Inc.) and a thermoplastic polymer, PCL have been chosen to study the dielectrostriction effect in oscillatory shear flow. The silicone elastomer was tested at oscillation frequency of $20 \mathrm{~Hz}$ and strain amplitudes of $10 \%, 15 \%, 20 \%, 25 \%$, and 30\%, respectively. Similarly, PCL was tested at oscillation frequency of $2 \mathrm{~Hz}$ and strain amplitudes of $2 \%, 3 \%, 4 \%, 5 \%$, and $6 \%$, respectively. The same measurement procedure was applied to the PCL sample at different temperatures, i.e., 110 and $120^{\circ} \mathrm{C}$. Equation (20) predicts that the dielectrostriction response is proportional to the square of the shear deformation, $\gamma^{2}$. For a shear oscillation, $\gamma=\gamma_{0} \sin \omega t$, the frequency of the dielectrostriction output signal is double that of the oscillations, i.e., $2 \omega$, and is proportional to square of the oscillation magnitude, $\gamma_{0}^{2}$. For all tests, both the total and the elastic shear stresses due to the circular annular Couette flow in the region (2), Fig. 6, are recorded simultaneously with the dielectrostriction output measured at double frequency of oscillations.

\section{Testing results}

\section{Silicone}

The dielectrostriction and the rheological responses of silicone elastomer are presented in Fig. 7. The rheometer produced oscillatory shear deformations having a frequency of $20 \mathrm{~Hz}$ and several values of the strain amplitudes: $10 \%, 15 \%, 20 \%, 25 \%$, and $30 \%$. The dielectrostriction signal $\sqrt{V}$, elastic shear stress, $\sigma^{\prime}$, [see Fig. 7(a)] and the total shear stress, $\sigma$, [see Fig. $7($ b) ] behave similarly with the strain amplitude. A single scaling coefficient fits the dielectrostriction signal, $\sqrt{V}$, and the elastic shear stress, $\sigma^{\prime}$, to the same master curve over the entire testing range. Similarly, a single coefficient is required to fit the dielectrostriction signal and the total shear stress, $\sigma$. The raw data from Fig. 7 

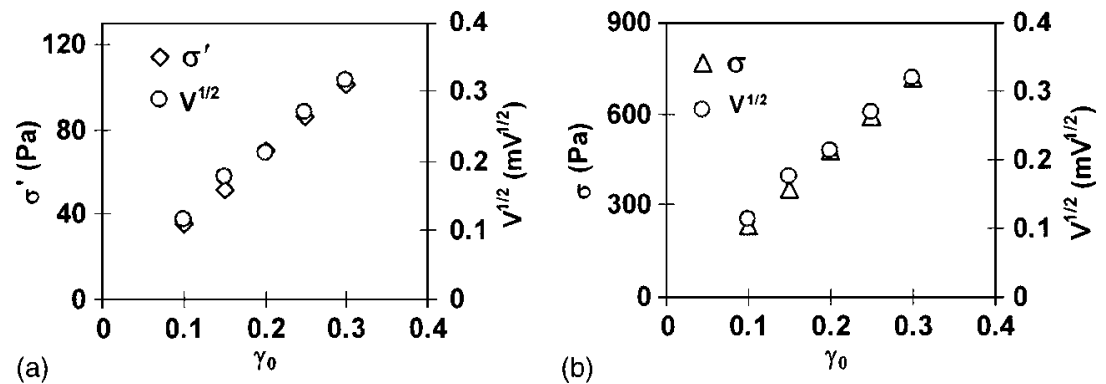

FIG. 7. Dielectrostriction and rheological responses in oscillatory shear flow of silicone at frequency, $f$ $=20 \mathrm{~Hz}$. The dielectrostriction output signal, $\sqrt{V}$, (a) elastic shear stress, $\sigma^{\prime}$, and (b) total shear stress, $\sigma$, behave similarly over the entire range of strain amplitude, $\gamma_{0}$.

are reploted in the form of dielectrostriction/stress relations in Figs. 8(a) and 8(b). The relationships between the shear dielectrostriction signal and the flow-induced shear stresses are linear.

\section{PCL}

The dielectrostriction and the rheological responses of PCL at $110^{\circ} \mathrm{C}$ during oscillatory shear flows at $2 \mathrm{~Hz}$ were obtained for the strain amplitudes of $2 \%, 3 \%, 4 \%, 5 \%$, and $6 \%$ and are presented in Fig. 9. The dielectrostriction signal, $\sqrt{V}$, elastic shear stress, $\sigma^{\prime}$, [see Fig. 9(a)] and the total shear stress, $\sigma$, [see Fig. 9(b)] behave similarly over the entire range of the strain amplitudes. In addition, the linear relationships between the dielectrostriction signal and flow-induced shear stresses exist, which are similar to those of silicone elastomer liquid. The same trend has been observed for PCL oscillatory shear flow under the same testing conditions except the temperature was changed to $120^{\circ} \mathrm{C}$. However, the constant coefficient between the dielectrostriction signal and the flowinduced stresses varies with the temperature, Figs. 10(a) and 10(b). The similar phenomenon has been reported in the stress-optic relationship [Janeschitz-Kriegl (1983)].

\section{Discussion}

Oscillatory shear deformation generates a shear component of the dielectric tensor which in coordinate system $X Y Z$ (see Fig. 1) can be expressed as
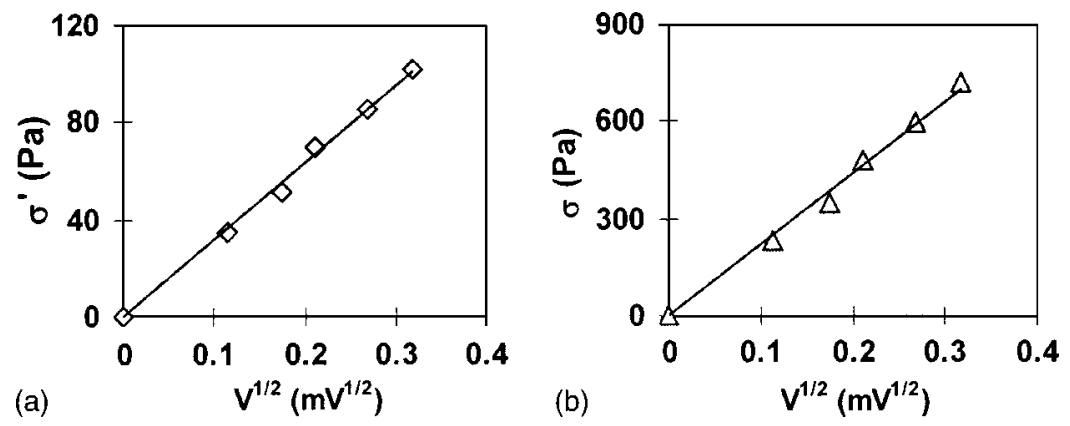

FIG. 8. The stress/dielectrostriction relationships for oscillatory shear flow of silicone at frequency $f$ $=20 \mathrm{~Hz}$ : both (a) elastic shear stress, $\sigma^{\prime}$, and (b) total shear stress, $\sigma$, are linear with dielectrostriction signal, $\sqrt{V}$. 

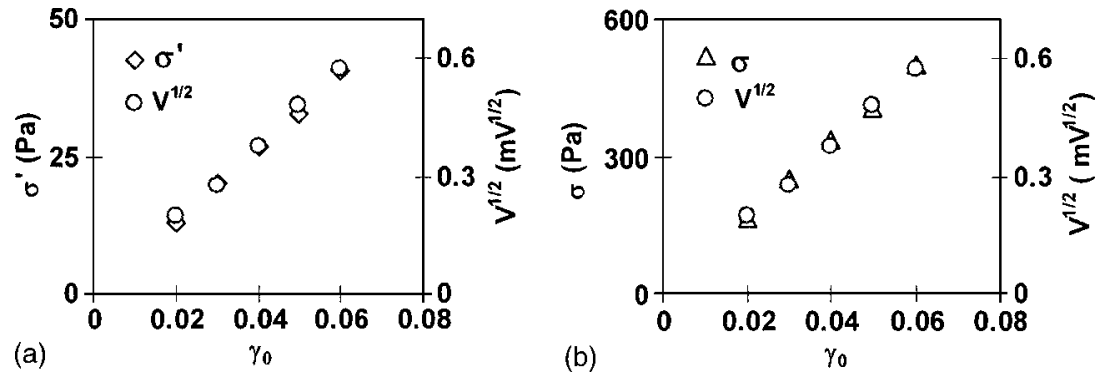

FIG. 9. Dielectrostriction and rheological responses for oscillatory shear flow of PCL at a frequency $f$ $=2 \mathrm{~Hz}$ and temperature $110^{\circ} \mathrm{C}$. The dielectrostriction signal, $\sqrt{V}$, (a) elastic shear stress, $\sigma^{\prime}$, and (b) total shear stress, $\sigma$, behave similarly over the entire range of strain amplitude, $\gamma_{0}$.

$$
\epsilon_{x y}=\alpha_{1} u_{x y}=\frac{\alpha_{1} \gamma_{0}}{2} \sin \omega t
$$

To obtain the constant, $C^{\prime}$, introduced by the SDR in Eq. (7), one needs to replace $\epsilon_{x y}$ using the earlier equation $\left(\epsilon_{x y}=\right) 1 / 2 \gamma_{0} \alpha_{1} \sin \omega t=C^{\prime} \sigma^{\prime} \sin \omega t$. Finally, the relationships between the constant, $C^{\prime}$, stresses and strains is

$$
C^{\prime}=\frac{\alpha_{1} \gamma_{0}}{2 \sigma^{\prime}} \propto \frac{\sqrt{V}}{\sigma^{\prime}} .
$$

The output signal of the planar capacitor sensor is proportional to square of the shear strain, $\gamma_{0}^{2}$, see Eq. (20). Both the elastic shear stress, $\sigma_{x y}^{\prime}$, and $\epsilon_{x y}$ component of the dielectric tensor are linear with the shear strains. Therefore, one can conclude that the SDR constant, $C^{\prime}$, is proportional to ratio of square root of the sensor voltage output, $\sqrt{V}$, and the stress, $\sigma_{x y}^{\prime}$. The experimental observations in Figs. 8(a) and 10(a) prove relation (22) and, therefore, are in agreement with the stress-dielectric rule formulated by Eq. (7). Our experimental data also show a linear stress-dielectric relationship between the total stresses and the dielectrostriction responses for all samples evaluated and for all testing conditions. The microscopic explanation of both stress-dielectric rules is yet to be
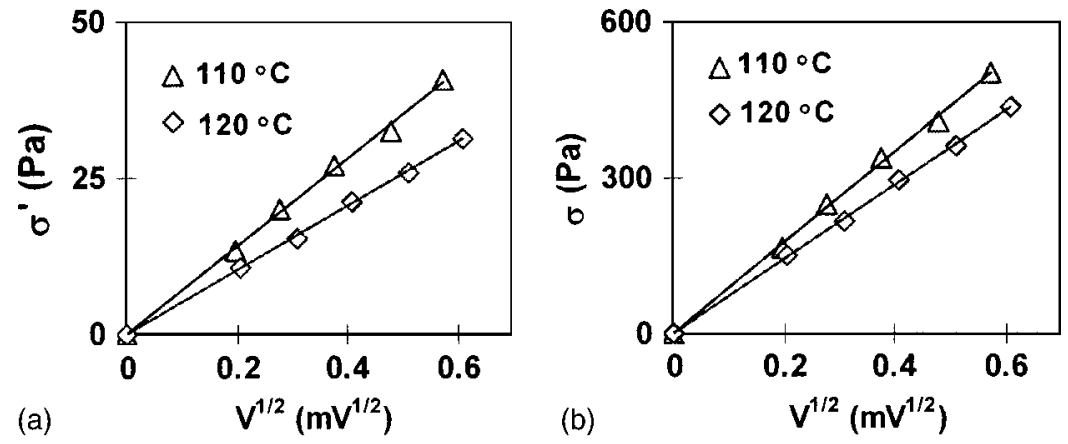

FIG. 10. Dielectrostriction and rheological responses of PCL in oscillatory shear flow at a frequency, $f$ $=2 \mathrm{~Hz}$ : both (a) elastic shear stress, $\sigma^{\prime}$, and (b) total shear stress, $\sigma$, are linear with dielectrostriction signal, $\sqrt{V}$, for temperatures 110 and $120^{\circ} \mathrm{C}$. However, the dielectric-stress coefficient (slope of the curves) varies with the temperature. 
finalized, and will be a subject of future studies. At this time we would like to point out that the linear relationship between shear dielectrostriction and the total flow-induced stresses has the same form as the well-established stress-optic rule

$$
\begin{gathered}
n_{x x}-n_{y y}=C\left(\sigma_{x x}-\sigma_{y y}\right), n_{y y}-n_{z z}=C\left(\sigma_{y y}-\sigma_{z z}\right), n_{z z}-n_{x x}=C\left(\sigma_{z z}-\sigma_{x x}\right) \\
n_{x y}=C \sigma_{x y}, n_{y z}=C \sigma_{y z}, n_{z x}=C \sigma_{z x} .
\end{gathered}
$$

The stress-optic rule holds well for many polymer melts and solutions because both the stress and the birefringence arise from the second moment of orientation of chain segments. The theoretical treatment of stress birefringence has been extensively studied. By considering the contribution of chain segments to the polarizability tensor and using the Lorentz-Lorenz equation to relate the refractive index and the polarizability, the second moment of the distribution of the end-to-end vectors of a system is proportional to the refractive index. On the other hand, molecular models also relate the stress tensor to the orientation tensor [Bird et al. (1987), Janeschitz-Kriegl (1983)].

Both the birefringence and the dielectrostriction involve polarization mechanisms and are related to the structure and orientation of the molecules. In addition, the flow-induced birefringence is the subset of the dielectrostriction which is observed in the optical range of the electromagnetic spectrum. Therefore, the existing birefringence models can be adopted to describe the dielectrostriction phenomenon if the local field effect can be neglected [Yamwong et al. (2002)]. The same orientation models and derivations for the stress birefringence can be employed to describe the contribution of the chain segments to the polarizability tensor. However, in some situations, such as liquid suspension, the local field effects could become prevalent and the disturbance field due to surrounding dipoles should then also be included.

At this stage, several questions related to stress/strain-dielectric relationship remain. First, the strain amplitude up to $30 \%$ is applied for dielectrostriction measurement in the oscillatory shear flow of a silicone elastomer. Our derivation assumes the measured dielectrostriction signal $V$ is proportional to the square of strain amplitude $\gamma^{2}$ in shear flow, where the higher order term $\left(\gamma^{4}\right)$ have been neglected. A shear strain of $30 \%$ corresponds to 0.09 in $\gamma^{2}$ and 0.0081 in $\gamma^{4}$, which might be considered as an estimate for the omitted terms. This value $(0.0081 / 0.09=9 \%)$ is close to the accuracy of our experimental measurements. However, the magnitude of the coefficient which multiplies $\gamma^{4}$ is unknown. At this stage, therefore, we do not know when the assumption about the small deformations is inadequate. Second, in the rheo-optic measurement, the stress-optic rule fails under large extensions due to the saturation of the orientational deformations of the polymeric chains. With stress-dielectric behavior, one more degree of freedom remains, namely uniaxial deformation of the chains. Therefore, one might also expect saturation of the stress-dielectric response but for much larger stresses. This, of course, awaits experimental verification.

Third, Eq. (2) expresses a first order expansion of the dielectric tensor with the respect to strains. Following Stratton (1941) and Landau, Lifshitz, and Pitaevskii (1984), we postulated the linear relation between the dielectric properties and strains. We have presently a fairly clear understanding of the parameters $\alpha_{1}$ and $\alpha_{2}$, their contribution to the total stress and/or the relation to microstructure. However, the test configuration proposed in this study provides a dielectric response which is quadratic with the strains. This suggests that Eq. (2) should be modified by including quadratic strain terms from the beginning, i.e.: 


$$
\Delta \epsilon_{i j}=\left(\epsilon_{i j}-\epsilon \delta_{i j}\right)=\alpha_{1} u_{i j}+\alpha_{2} u_{k k} \delta_{i j}+\beta_{1} u_{k k}^{2} \delta_{i j}+\beta_{2} u_{k k} u_{i j}+\beta_{3} u_{l m} u_{l m} \delta_{i j}+\beta_{4} u_{i l} u_{l j}
$$

This action introduces four more unknown coefficients: $\beta_{1}, \beta_{2}, \beta_{3}$, and $\beta_{4}$. These coefficients will not affect the orientation of principal axis of dielectric tensor but will redefine its principal values as indicated later

$$
\begin{gathered}
\epsilon_{1}=\epsilon+\alpha_{1}\left(\frac{\gamma^{2}}{4}+\frac{\gamma}{4} \sqrt{\gamma^{2}+4}\right)+\alpha_{2} \frac{\gamma^{2}}{2}+\beta_{2} \frac{\gamma^{2}}{2}+\beta_{4} \frac{\gamma^{2}}{4} \\
\epsilon_{2}=\epsilon+\alpha_{1}\left(\frac{\gamma^{2}}{4}-\frac{\gamma}{4} \sqrt{\gamma^{2}+4}\right)+\alpha_{2} \frac{\gamma^{2}}{2}+\beta_{2} \frac{\gamma^{2}}{2}+\beta_{4} \frac{\gamma^{2}}{4} \\
\epsilon_{3}=\epsilon+\alpha_{2} \frac{\gamma^{2}}{2}+\beta_{2} \frac{\gamma^{2}}{2} .
\end{gathered}
$$

All previous derivations remain valid but the final expression for capacitance will become

$$
\frac{\Delta C}{C_{0}}=\frac{C-C_{0}}{C_{0}}=\frac{2 \epsilon \alpha_{1}-\alpha_{1}^{2}+4 \epsilon \alpha_{2}+4 \epsilon \beta_{2}+2 \epsilon \beta_{4}}{8 \epsilon^{2}} \gamma^{2} .
$$

This will not alter the sensing application of the strain-dielectric phenomenon. However, a new experimental configuration and model study will be required to understand the implications of all parameters including the terms which are quadratic with strains.

\section{CONCLUSIONS}

The dielectrostriction effect and the linear relationship between the shear dielectrostriction response and the flow-induced stresses, called stress-dielectric rule, have been observed for both silicone elastomer and PCL liquids which were oscillated at constant frequencies. This rheological effect provides an avenue for novel sensing approaches to local monitoring stresses in polymeric flows. In addition, this capability might become a new tool for the study of material microstructure. The similarity between dielectrostriction and flow birefringence exists since both phenomena involve the polarization mechanism and are related to the structure and orientation of the polymeric chains; moreover, birefringence is the subset of dielectrostriction observed in the optical range of the electromagnetic spectrum. The existing birefringence models can be reformulated to describe the relationship between the dielectric constant and the microstructure of materials. However, when the local field effect is significant, the disturbance field due to the surrounding dipoles should be considered in the dielectrostriction models. At this stage, the stress/ strain-dielectric relationship in liquid polymers is still not fully understood and warrant further investigation.

\section{ACKNOWLEDGMENTS}

The authors thank Professor Rowlands for the helpful discussions. The authors greatly appreciate the issues raised by reviewers and the editor-the discussion section of the revised manuscript has been significantly improved and expanded in response to their comments. This research was supported in part by the AFOSR Grant No. FA9550-04-10238 and the 3M Company. 


\section{References}

Anderson, R. A., "Mechanical-stress in a dielectric solid from a uniform electric-field," Phys. Rev. B 33, 1302-1307 (1986).

Bird, R. B., C. F. Curtiss, R. C. Armstrong, and O. Hassager, Dynamics of Polymeric Liquids, Vol. 2, Kinetic Theory (Wiley-Interscience, New York, 1987).

Doeblin, E. O., Measurement Systems: Application and Design (McGraw-Hill, New York, 1990).

Filanc-Bowen, T. R., G. H. Kim, and Y. M. Shkel, "Shear and normal strain sensing with electroactive polymer composites," Smart Structures and Materials, Proc. SPIE, 3-6 March, 2003, San Diego, CA, 2003.

Holman, J. P., Experimental Methods for Engineers (McGraw-Hill, New York, 1994).

Horowitz, P., and W. Hill, The Art of Electronics (Cambridge University Press, London, 1998).

Janeschitz-Kriegl, H., Polymer Melt Rheology and Flow Birefringence (Springer, Berlin, 1983).

Landau, L. D., E. M. Lifshitz, and L. P. Pitaevskii, Electrodynamics of Continuous Media (Pergamon, New York, 1984).

Mase, G. T., and G. E. Mase, Continuum Mechanics for Engineers (CRC, Cleveland, 1999).

Nye, J. F., Physical Properties of Crystals (Oxford University Press, New York, 1985).

Saiz, E., and E. Rainde, Dipole Moments and Birefringence of Polymers (Prentice-Hall, Englewood Cliffs, NJ, 1992).

Shkel, Y. M., G. H. Kim, and R. E. Rowlands, "Analysis of graded composites fabricated by field-aided self-assembly techniques," International Symposium on Experimental Mechanics, Taipei, Taiwan, 2000.

Shkel, Y. M., and D. J. Klingenberg, "Material parameters for electrostriction,” J. Appl. Phys. 80, 4566-4572 (1996).

Shkel, Y. M., and D. J. Klingenberg, "Electrostriction of polarizable materials: Comparison of models with experimental data," J. Appl. Phys. 83, 7834-7843 (1998).

Shkel, Y. M., and D. J. Klingenberg, "A continuum approach to electrorheology," J. Rheol. 43, 1307-1322 (1999).

Stratton, J. A., Electromagnetic Theory (McGraw-Hill, New York, 1941).

Wales, J. S. L., The Application of Flow Birefringence to Rheological Studies of Polymer Melts (Delft University Press, The Netherlands, 1976).

Yamwong, T., A. M. Voice, and G. R. Davies, "Electrostrictive response of an ideal polar rubber," J. Appl. Phys. 91, 1472-1476 (2002). 